This item was submitted to Loughborough's Research Repository by the author.

Items in Figshare are protected by copyright, with all rights reserved, unless otherwise indicated.

\title{
Aluminoxane co-catalysts for the activation of a bis phenoxyimine titanium (IV) catalyst in the synthesis of disentangled ultra-high molecular weight polyethylene
}

\section{PLEASE CITE THE PUBLISHED VERSION}

http://dx.doi.org/10.1016/j.polymer.2015.07.039

\section{PUBLISHER}

(C) Elsevier

\section{VERSION}

AM (Accepted Manuscript)

\section{PUBLISHER STATEMENT}

This work is made available according to the conditions of the Creative Commons Attribution-NonCommercialNoDerivatives 4.0 International (CC BY-NC-ND 4.0) licence. Full details of this licence are available at: https://creativecommons.org/licenses/by-nc-nd/4.0/

\section{LICENCE}

CC BY-NC-ND 4.0

\section{REPOSITORY RECORD}

Romano, Dario, Efren Andablo-Reyes, Sara Ronca, and Sanjay Rastogi. 2015. "Aluminoxane Co-catalysts for the Activation of a Bis Phenoxyimine Titanium (IV) Catalyst in the Synthesis of Disentangled Ultra-high Molecular Weight Polyethylene". figshare. https://hdl.handle.net/2134/19720. 


\section{Aluminoxane Co-catalysts for the Activation of a Bis Phenoxyimine Titanium (IV) Catalyst in the Synthesis of Disentangled Ultra-High Molecular Weight Polyethylene}

Dario Romano*a ${ }^{\star a}$ Efren Andablo-Reyes ${ }^{a}$, Sara Ronca*a and Sanjay Rastogi ${ }^{a, b}$

*Corresponding authors: d.romano@lboro.ac.uk, s.ronca@lboro.ac.uk

a) Department of Materials, Loughborough University, Ashby Road, Loughborough, LE11 3TU, Leicestershire, UK

b) Research Institute, Teijin Aramid, Velperweg 76, Arnhem 6802ED, The Netherlands

\section{Abstract}

New activation systems for the bis[N-(3-tert-butylsalicylidene)pentafluoroanilinato] $\mathrm{Ti}$ (IV) dichloride catalyst (FI) for the synthesis of ultra-high molecular weight polyethylene (UHMWPE) with reduced entanglement density are explored. Together with the well-studied FI catalyst-methylaluminoxane (MAO) catalytic system, different aluminoxanes, namely polymethylaluminoxane-improved performance (PMAO), modified methylaluminoxane type 12 (MMAO12) and type 3A (MMAO3A) have been used. The catalyst activity increases with the addition of a co-catalyst modifier $(2,6$ di-tert-butyl-4-methylphenol, BHT). For an example when using MMAO3A as cocatalyst in combination with BHT the catalyst activity increases by tenfold. The synthesized UHMWPEs have been characterized via rheology, differential scanning calorimetry and uniaxial solid-state deformation to evaluate the influence that the different co-catalysts have on the catalyst activation and the entangled state of the polymer. Entanglement density increases when PMAO is used as a co-catalyst influencing the rheological response of the polymer melt and melting kinetics of nascent powder. Mechanical properties increase when MMAO12 is used in place of 
MMAO3A as co-catalyst. However, the earlier reported mechanical properties of UHMWPE synthesized using the FI/MAO are found to be better than those obtained using the co-catalysts investigated in this study.

\section{Introduction}

Single site olefin polymerization catalysts, in suitable reaction conditions, have been reported to produce Ultra-High Molecular Weight Polyethylene with a reduced number of entanglements [1]. This polymer, compared to those commercially available, shows improved solid-state processability and higher performance in terms of both, tensile modulus and strength in the uniaxially drawn products, resulting in an excellent candidate for its use in high demanding applications $[2,3]$. Studies performed on the influence of synthetic conditions on the properties of the material have shown that the entanglement density can be controlled to a certain extent. For example, by lowering the reaction temperature or the monomer partial pressure thus favoring the crystallization rate over the polymerization rate, it is possible to reduce the entanglement density. Moreover, since most of the entanglements are formed at the beginning of the reaction, the density of entanglements reduces with the synthesis time [4,5]. Studies along this line have been conducted in our research group using a bis(phenoxyimine) Titanium (IV) single site catalyst activated by a methylaluminoxane (MAO) co-catalyst $[6,7]$. This combination is considered one of the most active in ethylene polymerization [8] and has the additional advantage of being 'quasi-living' [9] (i.e. the molecular weight will increase with increasing the polymerization time in certain conditions) and producing a polymer with narrow molecular weight distribution: for these reasons, it is a good candidate to perform systematic studies on the influence that reaction conditions have on polymer properties.

Apart from the catalyst used, another component which has a considerable influence on the catalytic system performances is the catalyst activator (also called co-catalyst). Several co-catalysts are known to be used in the synthesis of polyolefins with single site catalysts [10], and they are selected on the basis of criteria such as the type of solvent used, the phase in which the reaction is carried 
out and the cost. Generally, after catalyst activation an ion pair between co-catalyst (anion) and catalyst (cation) is formed: the steric and electronic characteristics of the ionic couple formed have a dramatic impact on the system performance (i.e. catalyst activity) and the resulting polymer produced (i.e. molar mass). Among many, possibly the most used ones in combination with single site catalysts are aluminoxanes-based co-catalysts, the most common one being MAO, produced by controlled hydrolysis of trimethylaluminum (TMA) in a toluene solution. This class of co-catalyst has the advantage of playing a double role in the reaction medium: apart from the catalyst activation, it scavenges impurities that could lead to catalyst deactivation.

Since 1980, MAO is used as a catalyst activator [11], however its exact structure and composition are still not well understood. Several structures ranging from linear to complex cage structures have been proposed for example by Sinn [12], based on mass balances and phase separation experiments in the alkylaluminoxanes synthesis; other studies tend to attribute to MAO a cage structure, based on a combination of a tert-butylaluminoxanes crystallographically characterizable structure [13] and computational studies [14]. Figure 1 shows some examples of proposed structures for the MAO.
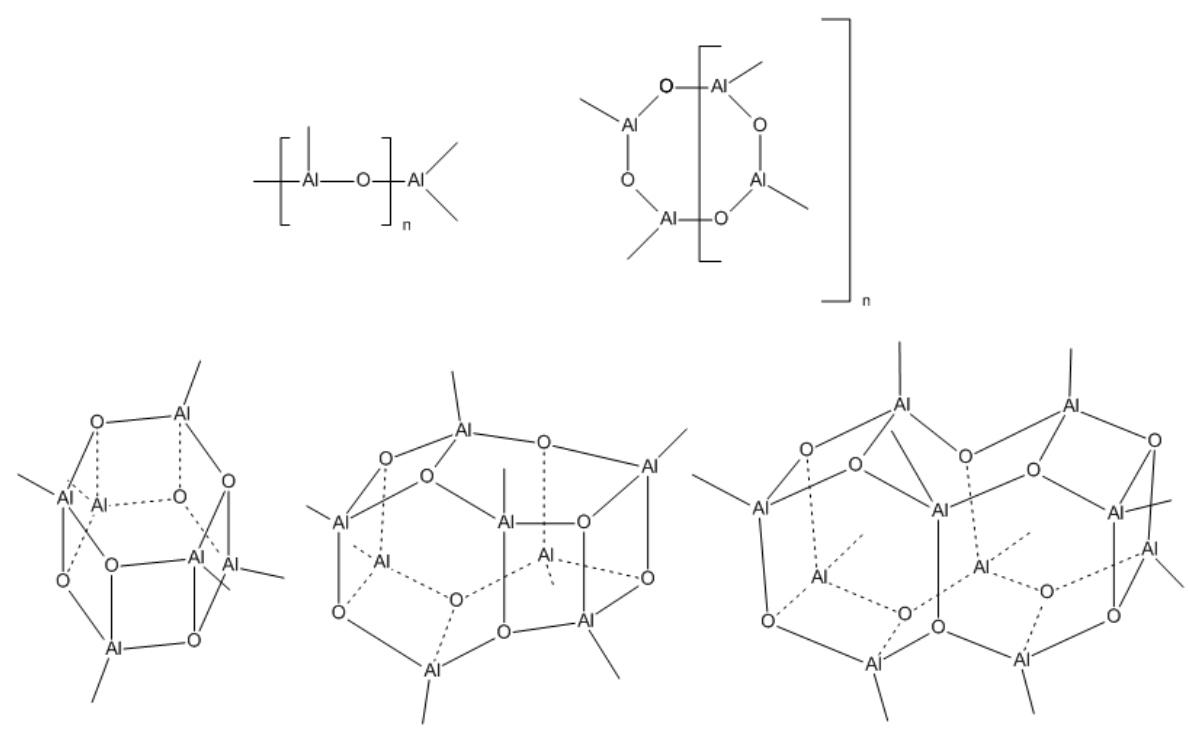

Figure 1 Selected structures proposed for the MAO. 
Compared with the other co-catalysts, such as alkylaluminums or small boranes, the MAO is able to stabilize the negative charge formed upon activation over a larger structure [15], thus resulting in a less 'sticky' ion couple that can accommodate the incoming monomer more easily [10]. Zurek and Ziegler showed for the catalytic system $\mathrm{Cp}_{2} \mathrm{ZrMe} \mathrm{H}_{2} / \mathrm{MAO}$ that the interaction between the MAO cage structure and the catalyst lead to dormant sites and catalytic active species showing the calculated energies [16].

Different aluminoxanes have been developed in order to produce activators that are soluble in different reaction media or that contain lesser amount of TMA. Similarly to MAO, the exact structure of these aluminoxanes is not known, and most probably is a combination of many. For example, the PolymethylaluminoxaneImproved Performance (PMAO) is synthesized using a non-hydrolytic process starting from different ketones and aldehydes compounds which can be alkylated by an alkylaluminum moiety [17]. Compared to MAO, the non-hydrolytic process possibly leads to a different cage structure(s). The PMAO has a molecular formula similar to $\mathrm{MAO},\left(\mathrm{CH}_{3} \mathrm{AlO}\right)_{\mathrm{n}}$. However, recent study suggests an exchange of methyl groups with higher alkyl groups [18]. Another difference in the co-catalysts is the alkylaluminum precursor. For example, the MMAO12 and MMAO3A are synthesized from bulkier trialkylaluminums (tri-n-Octylaluminum (TOAl) and tri-iso-butylaluminum (TiBAl) respectively). According to the molecular formulas for MMAO12 and MMAO3A $\left(\left[\left(\mathrm{CH}_{3}\right)_{0.95}\left(\mathrm{n}-\mathrm{C}_{8} \mathrm{H}_{17}\right)_{0.05} \mathrm{AlO}\right]_{\mathrm{n}}\right.$ and $\left(\left[\left(\mathrm{CH}_{3}\right)_{0.7}\left(\mathrm{i}-\mathrm{C}_{4} \mathrm{H}_{9}\right)_{0.3} \mathrm{AlO}\right]_{\mathrm{n}}[19]\right.$ respectively), the $n$-octyl and iso-butyl groups are likely to be placed on the external surface of the cage structures, necessarily not having the same structure. A common feature of all the aluminoxanes is the presence of a variable amount of TMA, used as a starting material in their synthesis.

Table 1 Content of trialkylaluminums in the different co-catalysts ${ }^{a}$.

\begin{tabular}{|l|l|l|l|l|}
\hline Co-catalysts & Albemarle & AkzoNobel & AkzoNobel & AkzoNobel \\
\hline
\end{tabular}




\begin{tabular}{|l|l|l|l|l|}
\hline & $\begin{array}{l}\text { (wt. \% of } \\
\text { TMA }\end{array}$ & $\begin{array}{l}\text { (wt. \% of } \\
\text { TMA })^{\star *}\end{array}$ & $\begin{array}{l}\text { (wt. \% of } \\
\text { TiBAl }\end{array}$ & $\begin{array}{l}\text { (wt. \% of } \\
\text { TOAl) }\end{array}$ \\
\hline MAO & 1.7 & -- & -- & -- \\
\hline PMAO & -- & $3.1-5.3$ & -- & -- \\
\hline MMAO12 & -- & $3.6-8.0$ & -- & $1.0-3.4$ \\
\hline MMAO3A & -- & $3.6-8.0$ & $3.5-10.7$ & -- \\
\hline
\end{tabular}

${ }^{a}$ It must be noticed that the concentration of trialkylaluminums can vary with the ageing and storing conditions.

* From the Certificate of Analysis as obtained from Albemarle

** Concentration taken from MSDS of the aluminoxanes

The presence of TMA has been proven to be detrimental for the performance of some single-site catalysts, especially the non-metallocene types, as it may act as a chain transfer agent (thus reducing the molecular weight of the polymer produced), or it may change the nature of the catalytic site leading to inactive species [20].

For example, in the case of the catalyst used for this study, the formation of dimethyl bridged complex is believed to occur, resulting in 'dormant' sites, as shown in Scheme 1 [15]. In accordance with the earlier findings no chain transfer to the TMA seems to occur when MAO is used as the activator [7].

Scheme 1 Interaction between the active site and the TMA. 


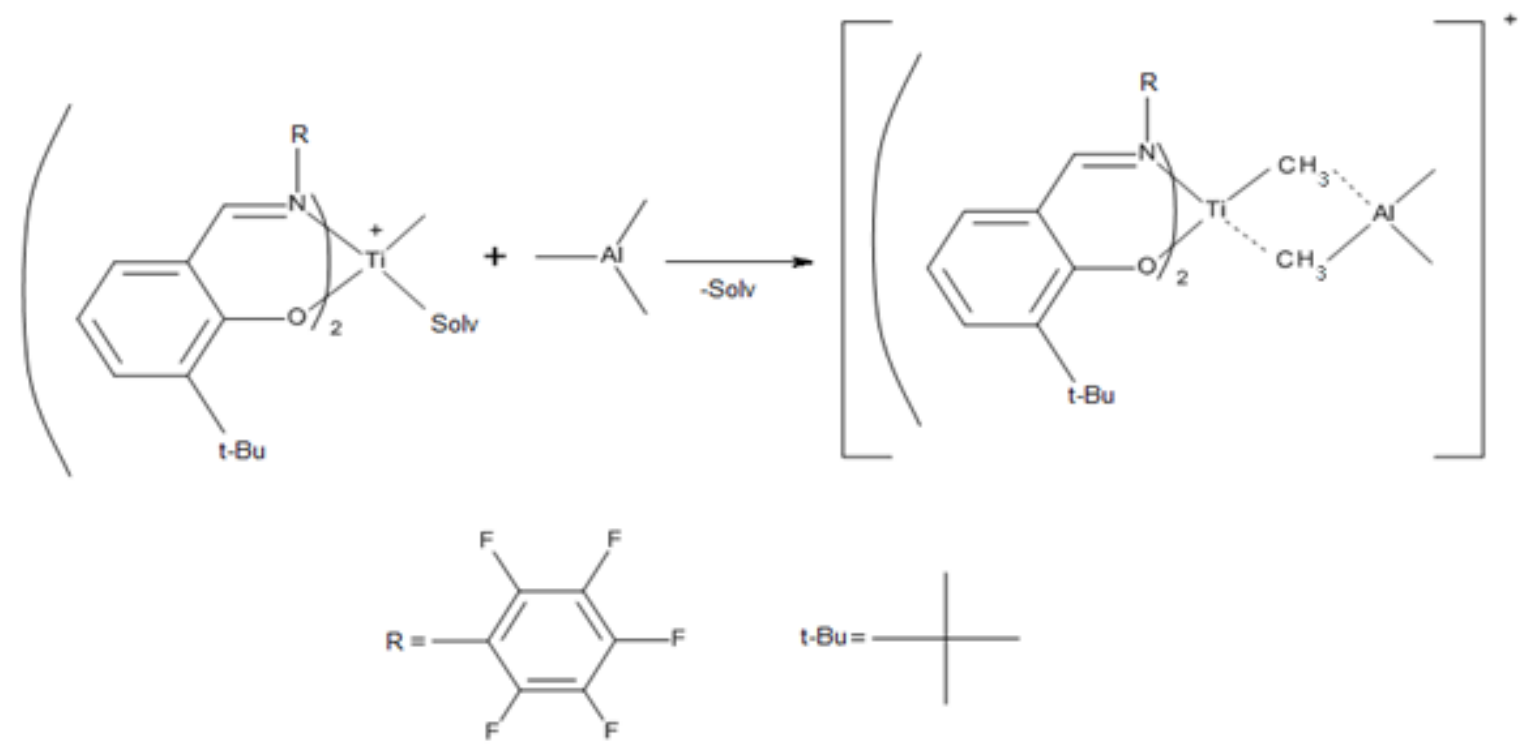

In order to prevent the deactivation processes, it is possible to remove the free TMA by drying the MAO solutions (also known as 'solid MAO' [21]) or to 'trap' it, by adding a hindered phenol, such as 2,6-di-tert-butyl-4-methylphenol (BHT), [6,7,20] that reacts with the free TMA to form sterically hindered aluminum-phenoxide species. In both cases higher catalytic activities are reported [7,21].

Alkylaluminums that are more hindered than TMA can also influence the catalytic performance: for example, it has been reported that the TiBAl can react with a bis(phenoxyimine)-type of catalyst leading to a complex that is inactive towards ethylene polymerization. The same catalyst used in this study has been tested by $\mathrm{Li}$ et al. in the presence of TiBAl and also in this case no catalytic activity is reported [22]. Because of the steric hindrance it is reasonable to think that bigger trialkylaluminums, in combination with the catalyst considered in this study, will preferentially not act as chain transfer agent. However, an interaction with the catalytic species cannot be ruled out.

Considering these facts it is evident that the performance of a co-catalyst is dependent on several factors. For example, the amount of active sites capable to generate, ion pair formed, the nature of trialkylaluminums present (in equilibrium with the polymeric form and/or on the surface of the aluminoxane), and on the cage structure. 
Following a preliminary study conducted in our research group [23], in this article we report a systematic study of the effect that different activators (MAO, PMAO, MMAO12 and MMAO3A) have on the activation of the bis[N-(3-tertbutylsalicylidene)pentafluoroanilinato] Titanium (IV) dichloride catalyst to promote the polymerization of ethene to UHMWPE while maintaining the disentangled state. The effect of a co-catalyst modifier, able to 'trap' the free TMA, is also studied. In all cases, reaction conditions are tailored to limit the formation of chain entanglements, so that the resulting UHMWPE produced retains the ease in processability [2]. The polymers produced are characterized by means of differential scanning calorimetry (DSC) and rheology, and the results obtained are compared to give a hypothesis on the role of the co-catalyst on the catalytic performance.

\section{Experimental}

\section{Materials}

All manipulations of air and moisture-sensitive compounds are performed under a nitrogen or argon atmosphere using standard high-vacuum Schlenk techniques or in a glovebox. Ethylene (grade 3.5) is purchased from BOC, bis[N-(3-tertbutylsalicylidene)pentafluoroanilinato] Titanium (IV) dichloride is received from MCat and used as received. Toluene (anhydrous 99.9\%) and 2,6-di-tert-butyl-4methylphenol ( $\geq 99.0 \%)$ are obtained from Sigma Aldrich ${ }$. Methylaluminoxane (10 wt. \% toluene solution) is purchased from Albemarle ${ }^{\circledR}$. PMAO, MMAO12 and MMAO3A are supplied from AkzoNobel. Irganox 1010 is supplied from BASF and is added to the polymer as antioxidant.

\section{Synthesis of UHMWPE}

Two different polymerization procedures are applied depending on the use of BHT.

An overnight pre-dried at $403 \mathrm{~K}$ cylindrical jacketed reactor vessel having a fivenecked flanged lid equipped with: nitrogen/ethylene feeding pipe tube, a 
thermometer probe holder, a stopper cone, gas release system and injection septum cone is backfilled with nitrogen after at least 3 cycles of vacuum/nitrogen. $750 \mathrm{ml}$ anhydrous toluene is introduced at room temperature into the reaction flask and dry nitrogen is bubbled through the solvent for few minutes under magnetic stirring.

\section{Polymerization with aluminoxanes solutions}

The desired amount of aluminoxane solution, used for scavenging the impurities from the system, is added. The reactor temperature is set at the desired value and controlled by a thermostat (Thermo Scientific NESLAB RTE7) connected to the thermometer probe. The nitrogen stream is then replaced by ethylene gas, and the solvent is saturated at the required partial pressure $\left(1.1\right.$ bar or $\left.1.1 * 10^{5} \mathrm{~Pa}\right)$. The pressure of ethylene is kept at the desired value by means of a Büchi gas flow-meter BPC6002. When the solvent saturation is reached at the desired temperature, the polymerization is initiated by addition of $5 \mathrm{mg}$ of the $\mathrm{FI}$ catalyst previously dissolved in $1 \mathrm{ml}$ of toluene and activated by the desired amount of aluminoxane solution in pre-contact for the required time.

\section{Polymerization with BHT-modified aluminoxanes solutions}

In the glove-box, the desired amount of $\mathrm{BHT}$ is dissolved in the aluminoxane solution and kept under stirring for 30 minutes. A desired portion of the previously prepared aluminoxane/BHT solution used for scavenging the impurities from the system is added. The reactor temperature is set at the desired value and controlled by a thermostat (Thermo Scientific NESLAB RTE7) connected to the thermometer probe. The nitrogen stream is then replaced by ethylene gas, and the solvent is saturated at the required pressure $\left(1.1\right.$ bar or $\left.1.1 * 10^{5} \mathrm{~Pa}\right)$. The pressure of ethylene is kept at the desired value by means of a Büchi gas flow-meter BPC6002. When the solvent saturation is reached at the desired temperature, the polymerization is initiated by addition of $5 \mathrm{mg}$ of the Fl catalyst previously dissolved in $1 \mathrm{ml}$ of toluene and activated by the remaining portion of aluminoxane/BHT solution. 
For both procedures, after the required polymerization time (60 min), the polymerization is quenched by the addition of acidified methanol. The resultant polymer is filtered, washed with copious amount of methanol/acetone and dried overnight in a vacuum oven at $313 \mathrm{~K}$. Irganox 1010 (0.7-1.0 wt. \%) is added to prevent oxidation during rheological experiments.

\section{Thermal Analysis}

Thermal characterization is performed in a differential scanning calorimeter Q2000, TA instruments, using nitrogen as purging gas at $50 \mathrm{ml} \mathrm{min}{ }^{-1}$. High precision TA Instruments T-zero pans and lids are used for all tests. Temperature and enthalpy calibration are performed using certified Indium. UHMWPE samples of $1.500 \pm 0.100$ mg are weighted on a Mettler-Toledo XS3DU precision balance (sensitivity of \pm $0.001 \mathrm{mg}$ ), and the pans are crimped with T-Zero lids. Heating-Cooling-Heating temperature ramps from 323 to $433 \mathrm{~K}$ are performed at a rate of $10 \mathrm{~K} \mathrm{~min}^{-1}$ in order to determine melting temperature and crystallinity. The annealing procedure is explained in details in ref. 5 .

As shown in previous studies [5,24], melting kinetics of UHMWPE that is dependent on entanglements between crystals in amorphous regions is followed by DSC. To recall, annealing of the nascent polymer powder at the temperature in the vicinity, but below the melting temperature, allows a clear distinction in melting response of the commercially available entangled samples and polymer synthesized to contain reduced entanglement density. The evolution of the linear integration of the enthalpy of fusion of the melt crystallized portion of crystals with respect to the total heat of fusion is used as a qualitative measure of the entanglement density in the nascent powder [5].

\section{$\underline{\text { Rheological Analyses }}$}

Linear rheology is a powerful technique to explore the dynamic response of viscoelastic polymer melt. Molecular characteristics from the linear viscoelastic 
frequency-dependent functions (Elastic $\left(G^{\prime}\right)$ and Viscous $\left(G^{\prime \prime}\right)$ moduli) are estimated by using a mathematical model. In entangled polymers, the well-established tube model (leading to the reptation theory) correlates the time-dependent relaxation modulus $(G(t))$ to molar mass of the components in the melt, as well as polymer network parameters, like molecular weight between entanglements $\left(M_{\mathrm{e}}\right)$. Talebi et al. [25] showed the strength of rheology over gel permeation chromatography in estimating weight-average molecular weight $\left(M_{\mathrm{w}}\right)$ and polydispersity index (PDI) of UHMWPEs having molar mass close to 9 million $\mathrm{g} / \mathrm{mol}$. The authors determined molar mass distribution of polymer chains by inverting the expression for the relaxation modulus given by the double reptation theory, equation 1 .

$$
G(t)=G_{N}^{0}\left(\int_{\ln M_{e}}^{\infty} F^{\frac{1}{2}}(t, M) w(M) d(\ln M)\right)^{2}
$$

In equation $1, G_{N}^{0}$ is the rubbery elastic plateau value, $w(M)$ represents the probability of finding chains with molar mass between $M$ and $M+d M, F(t, M)$ corresponds to the reptation memory function $F=\exp \left(-t / t_{d}\right)$, where the phenomenological relation for the reptation time $t_{d} \sim M^{3.4}$ has been used. Additionally, Pandey et al. [4] demonstrated that rheology could be used as a tool to estimate entangled state of the polymer obtained from the reactor. Both methodologies are employed to characterize the polymers synthesized in this work.

In order to perform rheological characterization the nascent powder is compressed into a plate of $35 \mathrm{~mm}$ diameter and thickness $0.6-0.7 \mathrm{~mm}$ at a fixed temperature of $398 \mathrm{~K}$, under a maximum force of 20 tons for an average time of 20 minutes. Using a punching device, $12 \mathrm{~mm}$ diameter discs are cut for rheological studies. Rheological experiments are performed using a $12 \mathrm{~mm}$ parallel plates strain controlled rheometer [TA instruments, ARES LS2]. The sample is placed between parallel plates at an initial temperature of $383 \mathrm{~K}$. To prevent oxidation, the temperature is controlled by a convection oven under nitrogen environment. After stabilization at $383 \mathrm{~K}$, temperature is increased to $403 \mathrm{~K}$ at $30 \mathrm{~K} / \mathrm{min}$. After thermal stabilization the sample is heated further to $433 \mathrm{~K}$ at $10 \mathrm{~K} / \mathrm{min}$ while maintaining the compression force of $4 \mathrm{~N}$. Subsequently, an oscillatory amplitude sweep test is carried out to determine the range of oscillatory strains in the linear viscoelastic region. The test is performed at a fixed frequency $(\omega)$ of $10 \mathrm{rad} / \mathrm{s}$. To follow changes 
in the elastic modulus of the polymer melt, an oscillatory time sweep is performed until no significant changes are observed [1]. The experiments are performed at a fixed frequency of $10 \mathrm{rad} / \mathrm{s}$ and a strain of $0.3 \%$, well within the linear viscoelastic regime.

As shown in ref [7], following the procedure of annealing the polymer in the melt state as described above, polymers synthesized under similar conditions are not able to achieve the thermodynamic stable melt state. Since achieving this thermodynamic stable melt state is a requisite for molar mass determination, a different procedure is applied. For this purpose that promotes entanglement formation without compression, the nascent UHMWPE powder (expected to have the molar mass above 6 million $\mathrm{g} \mathrm{mol}^{-1}$ ) is pre-annealed in a vacuum oven for 48 hours at $433 \mathrm{~K}$. Subsequently, the annealed powder is compression moulded at $433 \mathrm{~K}$. From the moulded plate, discs of $12 \mathrm{~mm}$ are obtained. Frequency sweep tests are performed in a range of 100 to $1 \times 10^{-3} \mathrm{rad} / \mathrm{s}$, at a constant strain within the linear viscoelastic response of the polymer, typically $0.5 \%$. Following the procedure described in ref [25], by performing linear step strain tests the frequency range is extended to values as low as $10^{-4} \mathrm{rad} / \mathrm{s}$. The time-dependent relaxation modulus is Fourier transformed to angular frequency space and used as complementary data for the oscillatory frequency sweeps in the low frequency range $\left(10^{-3}\right.$ to $\left.10^{-4} \mathrm{rad} / \mathrm{s}\right)$. Using the Orchestrator software available on the rheometer, $M_{\mathrm{w}}$ and PDI are determined by means of the method developed by Mead [26].

\section{Mechanical characterization procedures}

\section{Shaping of the synthesized powder to uniaxially oriented tapes}

For determination of the mechanical properties of the uniaxially deformed disentangled UHMWPE the molding of nascent powder without melting is desired. For compression molding of the powder without melting, $25 \mathrm{~g}$ of polymer powder is poured into a mold having cavity of $620 \mathrm{~mm}$ in length and $30 \mathrm{~mm}$ in width. The uniformly dispersed low density powder in the mold is compression-molded at $129{ }^{\circ} \mathrm{C}$ and 130 bar for $10 \mathrm{~min}$. The resulting sheet of $1.42 \mathrm{~mm}$ thickness is 
preheated for at least $1 \mathrm{~min}$ at a constant temperature of $136{ }^{\circ} \mathrm{C}$ and rolled with a Collin calender (diameter rolls: $250 \mathrm{~mm}$, slit distance $0.15 \mathrm{~mm}$, inlet speed 0.5 $\mathrm{m} / \mathrm{min}$ ). While rolling (speed $2.5 \mathrm{~m} / \mathrm{min}$ ) the sheet is stretched partially. The rolled and stretched sheet is further stretched in two steps on a $50 \mathrm{~cm}$-long, oil heated hot plate. The draw ratio is obtained by dividing the specific weight of the sheet prior to deformation by the specific weight of the tape after stretching. A typical processing temperature of polyethylene in the two stretching steps ranges between $130{ }^{\circ} \mathrm{C}$ to $154{ }^{\circ} \mathrm{C}$. The higher stretching temperature, above $140{ }^{\circ} \mathrm{C}$, is used for the partially stretched samples where the macroscopic forces can be transferred to molecular level under external constraint. To recall, melting temperature of linear uniaxially stretched UHMWPE can be increased under external constraints. The sample is stretched to the desired initial draw ratio in the first stretching step. Parts of the drawn sample are used to measure the mechanical properties, whereas the remainder of the sample is drawn further to the final draw ratio and the mechanical properties are determined subsequently.

\section{Determination of the mechanical properties}

Tensile properties were measured according to ASTM D7744-2011 using an Instron 5566 tensile tester at room temperature $\left(25^{\circ} \mathrm{C}\right)$. To avoid any slippage, the side action grip clamps with flat jaw faces are used. The nominal gauge length of the specimen is $100 \mathrm{~mm}$, and the test is performed at a constant rate of extension (crosshead travel rate) of $50 \mathrm{~mm} / \mathrm{min}$. The breaking tenacity (or tensile strength) and modulus (segment between 0.3 and $0.4 \mathrm{~N} / \mathrm{Tex}$ ) are determined from the force against displacement between the jaws.

\section{Results and discussion}

\section{Ethylene polymerization}

Polymerization reactions are carried out at controlled temperature of $283 \mathrm{~K}$, with a maximum fluctuation of $10 \mathrm{~K}$ due to the exothermal nature of the reaction. This 
deviation occurs at the initial phase of the reaction and is usually dissipated in approximately 2 minutes. Table 2 summarizes the ethylene polymerization results.

Table 2 Ethene polymerization results.

\begin{tabular}{|c|c|c|c|c|c|c|c|c|c|}
\hline Run & Co-catalyst & $\begin{array}{l}\text { BHT } \\
\text { (g) }\end{array}$ & $\begin{array}{l}\text { Yield } \\
\text { (g) }\end{array}$ & $R p^{a}$ & $M_{\mathrm{w}}^{b, c}$ & $M_{\mathrm{n}}^{\mathrm{b}, \mathrm{c}}$ & $\mathrm{PDI}^{\mathrm{C}}$ & $\begin{array}{l}T_{m} \\
(K)\end{array}$ & $\begin{array}{l}\text { Crys. }^{d} \\
(\%)\end{array}$ \\
\hline 1 & MAO & 0 & 27 & $4.0 * 10^{3}$ & 9.0 & 2.6 & 3.4 & 411.6 & 81 \\
\hline 2 & $\begin{array}{l}\text { MAO } \\
+\mathrm{BHT}\end{array}$ & 1.00 & 35 & $4.9 * 10^{3}$ & 7.2 & 2.7 & 2.7 & 413.3 & 77 \\
\hline 3 & PMAO & 0 & 20 & $2.9 * 10^{3}$ & 10.7 & 3.0 & 3.6 & 412.7 & 79 \\
\hline 4 & $\begin{array}{l}\text { PMAO } \\
+\mathrm{BHT}\end{array}$ & 1.18 & 26 & $3.9 * 10^{3}$ & 10.3 & 2.8 & 3.7 & 411.9 & 77 \\
\hline 5 & MMAO12 & 0 & 23 & $3.3 * 10^{3}$ & 7.1 & 1.1 & 6.7 & 412.2 & 74 \\
\hline 6 & $\begin{array}{l}\text { MMAO12 } \\
+\mathrm{BHT}\end{array}$ & 2.90 & 30 & $4.3 * 10^{3}$ & 5.0 & 2.5 & 2.4 & 412.2 & 77 \\
\hline 7 & MMAO3A & 0 & 3 & $4.0 * 10^{2}$ & n.d. & n.d. & n.d. & 413.3 & 73 \\
\hline 8 & $\begin{array}{l}\text { MMAO3A } \\
+\mathrm{BHT}\end{array}$ & 1.57 & 30 & $4.5^{\star} 10^{3}$ & 8.5 & 2.8 & 3.0 & 412.3 & n.d. \\
\hline
\end{tabular}

Other Conditions: Al/Ti molar ratio, 1200 when MAO is used; 2600 for PMAO, MMAO12 and MMAO3A; Catalyst, $6.2 \pm 0.2 \mu \mathrm{mol}$; Reaction solvent, $750 \mathrm{ml}$ of toluene; Reaction time, $60 \mathrm{~min}$; Ethylene Pressure, 1.1 bar $\left(1.1 * 10^{5} \mathrm{~Pa}\right)$; Temperature, $283 \mathrm{~K}$. ${ }^{\text {a }}$ Catalytic activity in

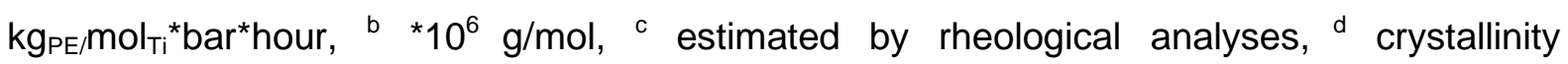
calculated from the melting enthalpy measured by DSC experiments and related to the theoretical value for $100 \%$ crystalline polyethylene, $293 \mathrm{~J} / \mathrm{g}$ [27].

MAO used in combination with BHT was demonstrated to be the most efficient co-

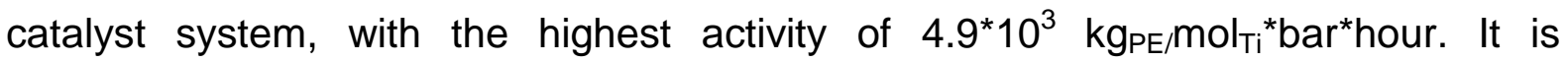
important to consider that the amount of MAO needed to activate the catalyst is less than half compared with the rest of the co-catalysts used. The catalytic system, 
formed by the combination of FI catalyst and MMAO3A, has an activity of one order of magnitude lower compared to the rest of catalytic systems studied here. In agreement to previous finding [22], this result suggests that the catalyst can be modified by the TiBAl (which is present in the MMAO3A solution), leading to nonactive species toward ethylene polymerization.

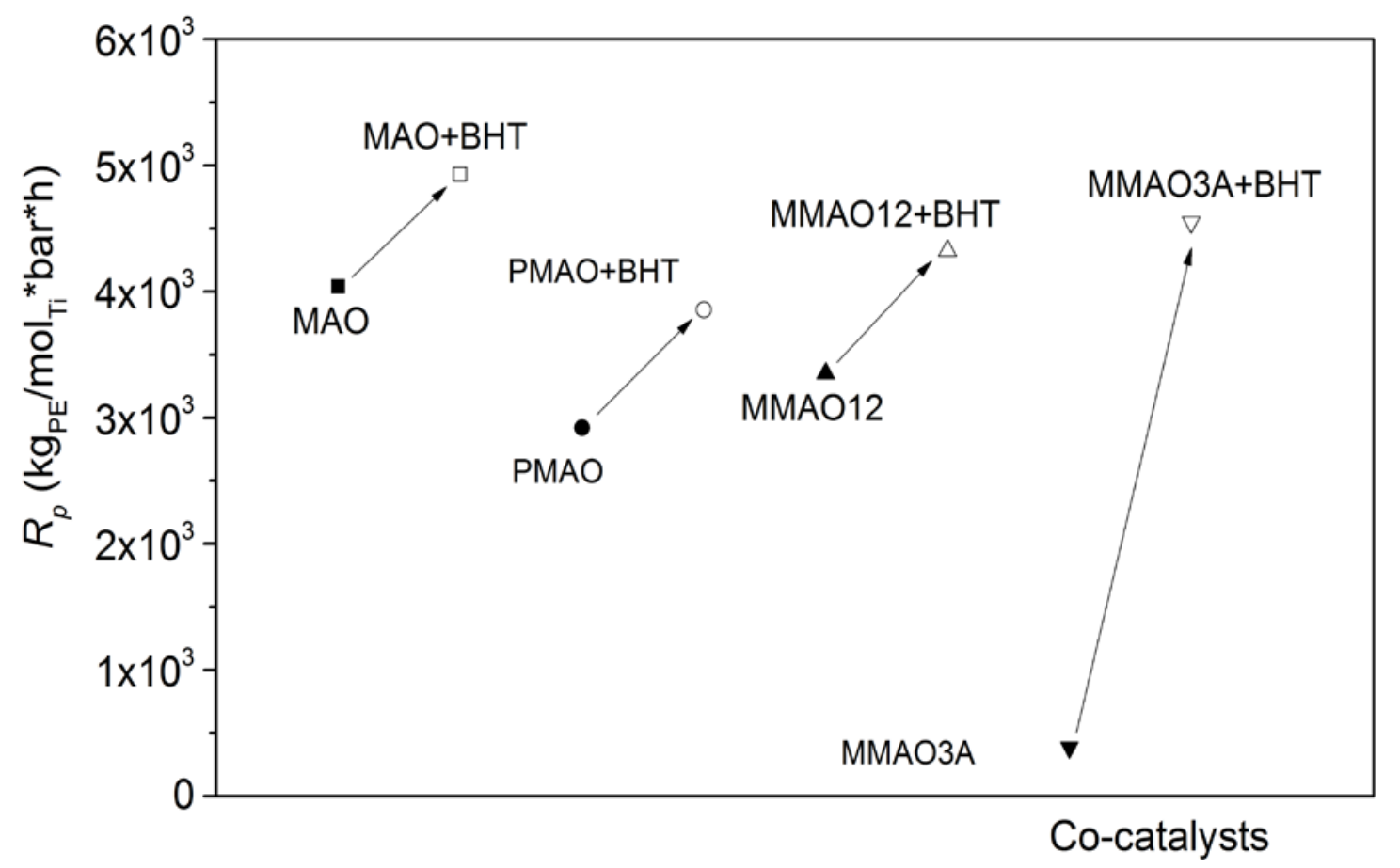

Figure 2 Catalyst activity as a function of the different co-catalysts: filled symbols represent the value of catalyst activity when the catalyst is activated with the aluminoxanes only; unfilled symbols represent catalyst activity when the BHT is used.

The low yield of the run 7 leads to high catalytic ashes content and the rheological response of the polymer was affected. For this reason the characterization of this polymer is excluded in the discussion.

For all the co-catalysts tested, the effect of the BHT is an enhancement of the catalyst activity (figure 2). This is particularly striking in the case of MMAO3A: when this is used in combination with BHT, the catalyst activity increases up to $4.5^{\star} 10^{3}$

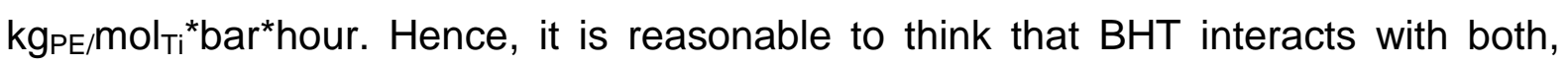


TMA and TiBAl, trapping them and preventing their damaging influence on the catalyst.

\section{$\underline{\text { Rheological analyses }}$}

On melting UHMWPE with reduced number of entanglements, a non-equilibrium melt state is obtained in the linear viscoelastic region having the elastic shear modulus $G^{\prime}$ lower than the value expected in the equilibrium melt state $\left(G^{\prime} \sim 2.0\right.$ MPa). In the case of UHMWPE having molar mass up to 6 million $\mathrm{g} / \mathrm{mol}$, $G^{\prime}$ increases monotonically until it reaches the equilibrium value in a time interval that rescales with the $M_{\mathrm{w}}$ approximately as $M_{\mathrm{w}}{ }^{2.4}$ [7]. Systematic studies performed by Pandey et al. [4] showed that the initial storage modulus reflects the entangled state of the polymer formed during polymerization. Higher the starting modulus, higher will be the entanglement density created during polymerization. The elastic modulus build-up is shown in figure 3 for all the polymers of table 2. During measurement, the temperature is kept constant at $433 \mathrm{~K}$, approximately $20 \mathrm{~K}$ above the equilibrium melting temperature of polyethylene. To allow thermal stabilization in the rheometer, the data collection is started after 90 seconds the set temperature is reached. Absolute values of $G^{\prime}$ at the start of the build-up are in a range between 0.4 and 0.8 $\mathrm{MPa}$, lower than at least half the value expected for a UHMWPE melt in equilibrium. Following the reasoning in ref. 4 it can be concluded that the polymer powder, as coming from the reactor, contains low entanglement density. Despite the proximity of initial values of $G$ ' for the polymers synthesized, some differences are evident. Considering the starting modulus, polymer synthesized using MAO and MMAO12 appears to have lesser density of entanglements compared to polymer synthesized using PMAO as co-catalyst. The use of $\mathrm{BHT}$ as co-catalyst modifier helps decreasing the density of entanglements in the case of the three co-catalysts previously mentioned. The synthesis using MMAO3A is successful only when the cocatalyst is used in combination with BHT. However, it is apparent that the polymer synthesized using MMAO3A+BHT displays the larger entanglement density compared to the rest of polymer studied here. In figure 3 , the modulus build-up is followed for $1.8 \times 10^{5}$ seconds. In this period of time, only the polymer synthesized 
using MMAO3A+BHT as co-catalyst reaches closest to the equilibrium value for $G^{\prime}$ during the given experimental time.

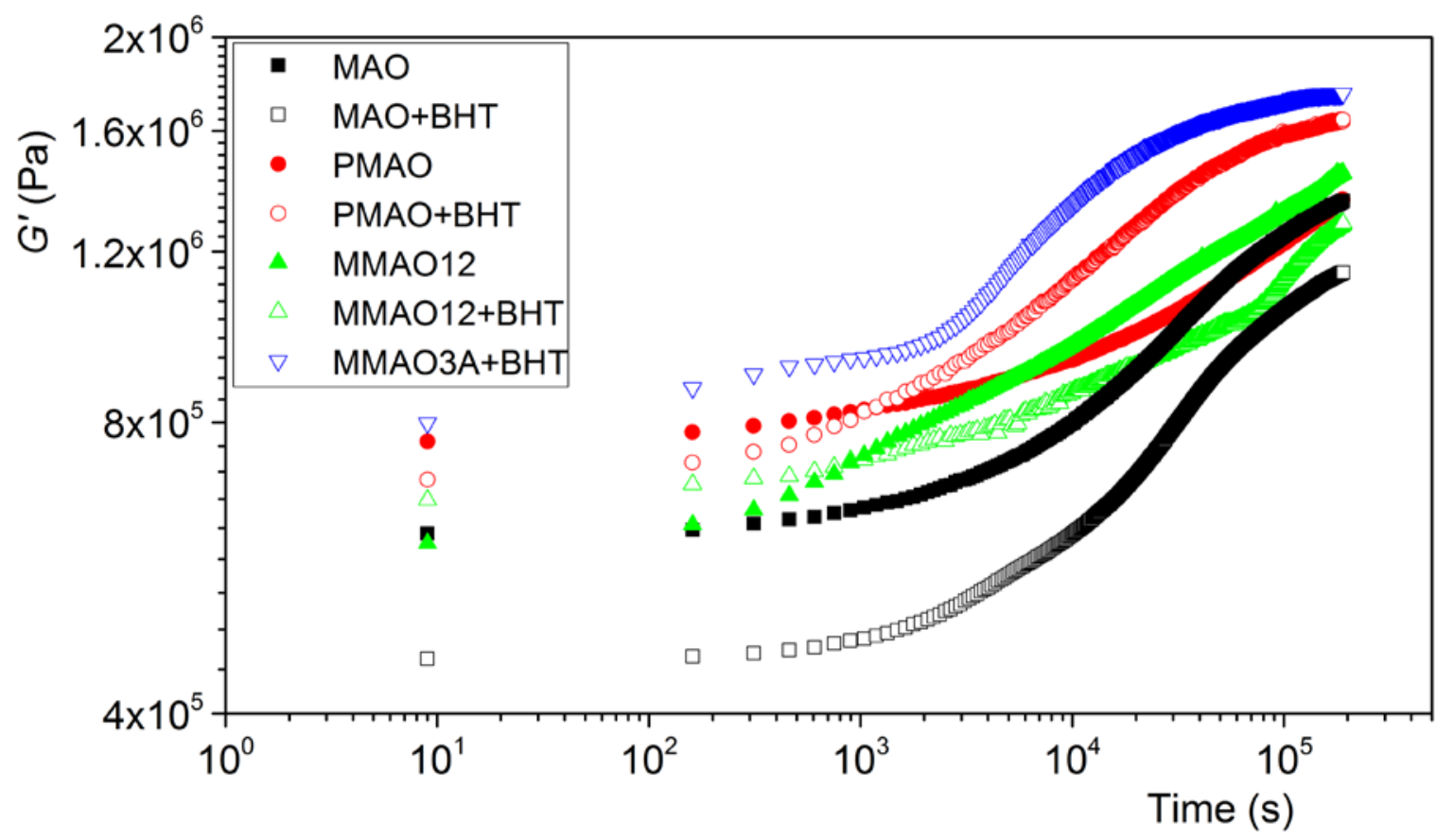

Figure 3 Elastic modulus build-up for polymer synthesized using the different catalytic systems. The low values of the elastic modulus $G$ ' indicate the low density of entanglements in the nascent polymer powder.

In order to apply consistently the tube model for molar mass determination, it is necessary to achieve a polymer melt in the equilibrium state. As mentioned in the experimental section, on applying an annealing step at a constant temperature of $433 \mathrm{~K}$ under vacuum for 48 hours prior to the rheological characterization, the equilibrium state is reached for all polymers studied here in a range of 20 to 36 hours.

Two equivalent linear rheological experiments, step strain relaxation and oscillatory frequency sweep, are performed on the thus obtained thermodynamically equilibrated melt. The time-dependent relaxation modulus is obtained from the former, while from the latter, two frequency-dependent functions known as elastic $G$ ' and viscous G" modulus are obtained. The functions obtained from these methods are co-related via a Fourier transformation. 
In order to illustrate the influence of molecular mass on the linear rheological response of the melt, $G(t)$ are presented in figure 4 for two different polymers synthesized using MMAO12+BHT (unfilled symbols) and MMAO3A+BHT (filled symbols). The tube model describes the stress relaxation by chain reptation as an exponential time decaying function. The decaying time, known as reptation time, has been found to be proportional to $M_{\mathrm{w}}{ }^{3}$ for these high molar masses, which is in agreement with the reptation theory $[28,29]$. Bearing this in mind, and considering the differences in the anticipated cross-over points from the stress relaxation moduli presented in figure $4 \mathrm{a}$, it is evident that the polymer synthesized using MMAO3A+BHT has a higher $M_{\mathrm{w}}$ compared to the one synthesized using MMAO12+BHT.

In order to determine numerical values of molar mass with the aid of the method developed by Mead and commercialized by TA instruments, it is necessary to obtain the viscoelastic functions. As explained above, these functions are directly obtained by a small amplitude oscillatory test. The rheometer employed in this work is able to cover a range in oscillatory mode from 600 to $1 \times 10^{-3} \mathrm{rad} / \mathrm{s}$. As explained before, $G(t)$ obtained from step strain experiments is converted to the frequency space, allowing to extend this range to values as low as $1 \times 10^{-4} \mathrm{rad} / \mathrm{s}$, facilitating a better estimation of large molar mass components. Viscoelastic functions obtained by both methods are shown in figure 4. A good overlap is observed in the broad frequency range. The difference related to the molar mass of components is evident at low frequencies, since relaxation in the frequency domain is observed as the transition from predominantly elastic to predominantly viscous behavior, $G$ " becomes larger than $G$ ' after the crossover point at low frequency. As observed in the tendency of the curves in figure $4 \mathrm{~b}$, this process will occur first for the polymer synthesized using MMAO12+BHT compared to the one synthesized using MMAO3A+BHT. 

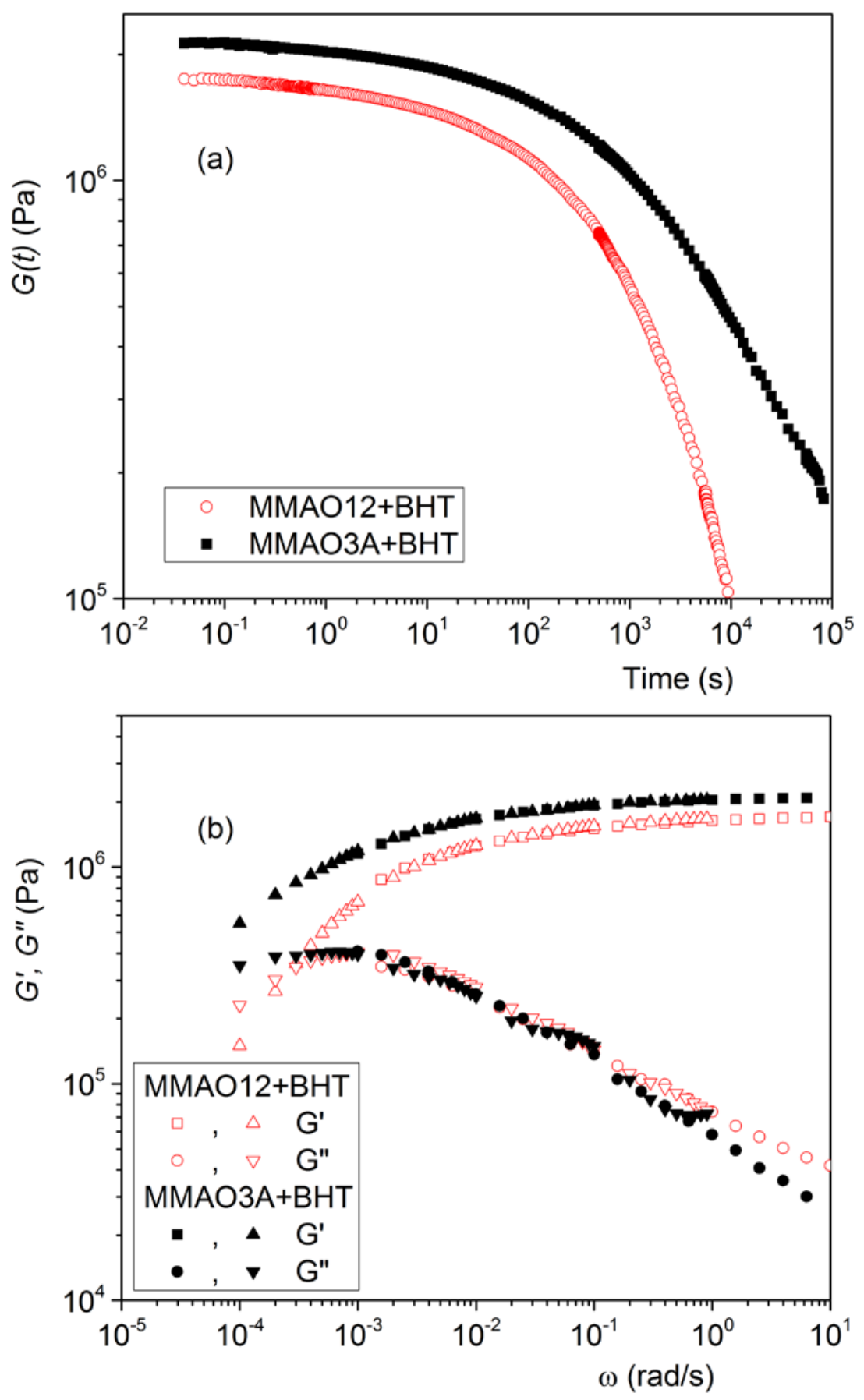

Figure 4 (a) $G(t)$ as a function of time and (b) G' and $G$ " as a function of frequency for the polymers synthesized using MMAO12+BHT (unfilled circles) and MMAO3A+BHT (filled squares) as co-catalysts. Differences in the viscoelastic functions immediately suggest larger molar mass content in the polymer synthesized using MMAO3A+BHT compared to the one synthesized using MMAO12+BHT. The triangles (up and down, filled and unfilled) are obtained from the stress-relaxation experiments. 
The viscoelastic functions are inverted using the reptation model to obtain the distribution function. The molar mass of the melt components is assumed to follow a Shulz-Flory distribution. From the distribution function, $M_{\mathrm{w}}$ and PDI are calculated and presented in table 2 . The polymers synthesized using all the catalytic systems have $M_{\mathrm{w}}$ greater than or equal to $5 * 10^{6} \mathrm{~g} / \mathrm{mol}$. This confirms the ability of this catalytic system to synthesize UHMWPEs under the polymerization conditions established in this work. In the case of polymers synthesized using PMAO (also in combination with $\mathrm{BHT}$ ) the resulting molecular weights are slightly higher compared to the molecular weight obtained using MAO, MMAO12 and MMAO3A in combination with BHT.

Studies on polymers synthesized using MAO, PMAO and MMAO12 show that the addition of BHT in the activation causes a slight decrease in the $M_{w}$ (Figure 5). This is apparently in contrast with previous findings that described the use of BHT as a way to prevent the chain transfer process that lowers the molecular weight [20]. In a previous study, the authors suggested that second species may be present when there is free TMA, which is capable to produce chains with higher molecular weights [7]. Considering that a certain amount of TMA is always present, presence of the second specie is likely to occur when other co-catalysts are used, (see table 1). The requirement of higher $\mathrm{Al} / \mathrm{Ti}$ ratio for the $\mathrm{PMAO}, \mathrm{MMAO} 12$ and MMAO3A in order to have comparable yields, but resulting in polymers of similar molecular weight, possibly suggests that these co-catalysts have a lower catalyst activation capability compared to MAO. 

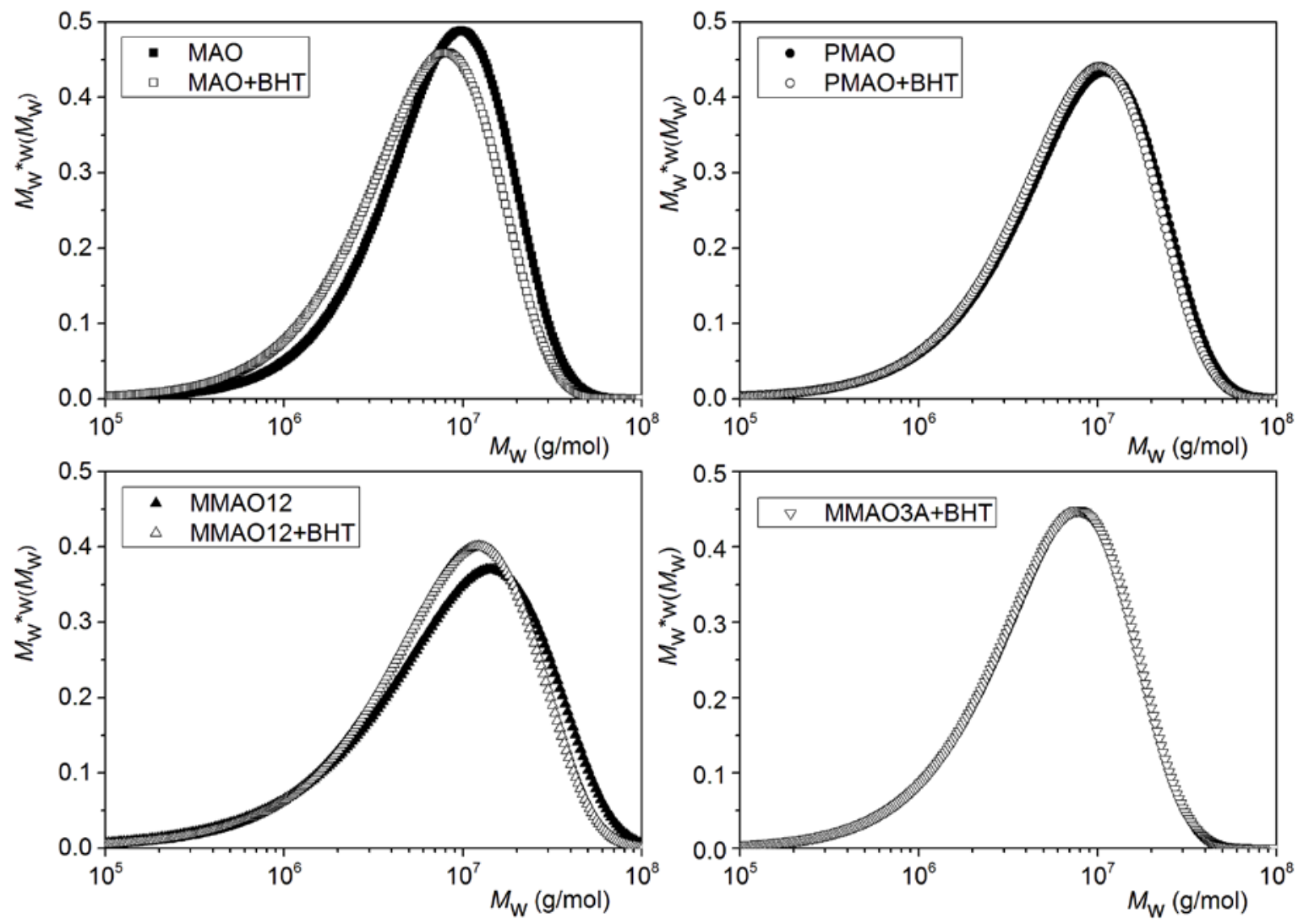

Figure 5 Distribution function for polymers synthesized using the different co-catalysts and in combination with BHT.

\section{DSC analysis for the estimation of entanglements in reactor powders}

Polymers produced using the co-catalysts MAO, PMAO and MMAO12 with and without $\mathrm{BHT}$ show a well-defined melting peak with nominal melting temperature about $412 \mathrm{~K}$ during the first heating ramp. This melting temperature is characteristic of nascent HDPE/UHMWPE. Crystalline contents estimated from the enthalpy of fusion are in the range of 73 up to $81 \%$.

The presence of entanglements in the amorphous region of the semi-crystalline nascent powder is investigated by annealing experiments, as described in the experimental section. Different annealing times are chosen in a range from 15 minutes up to 24 hours. In this duration the temperature is fixed at a value calculated as explained in ref 5 . Figure 6 shows the typical evolution of the melt phase 
transition, as seen by DSC, of the previously annealed polymer for different annealing times.

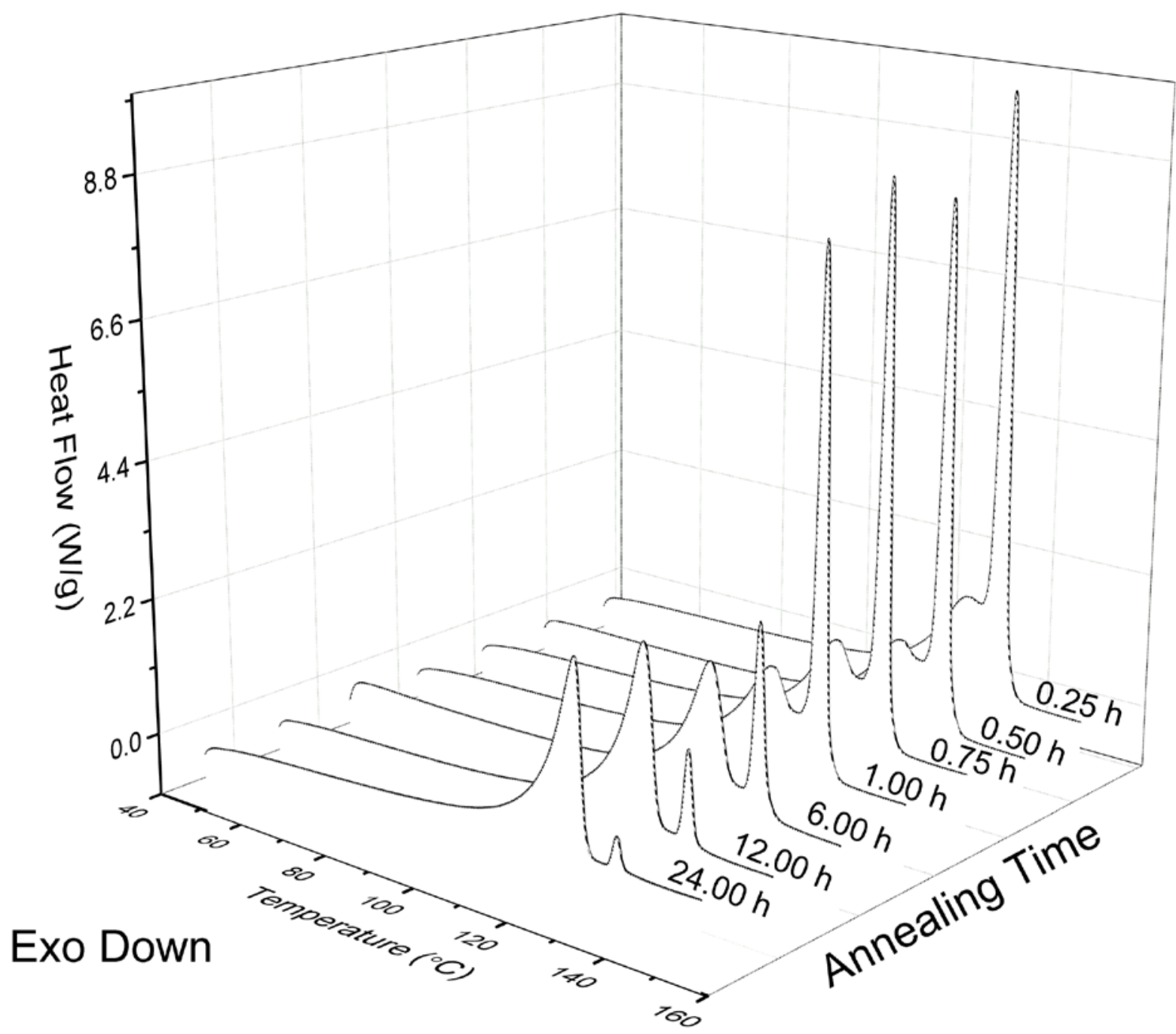

Figure 6 Evolution of the melting peaks after annealing at $411.3 \mathrm{~K}$ for different annealing time for the polymer synthesized using MAO as co-catalyst.

In figure 6 , the melting peak at low temperature, approximately $407 \mathrm{~K}$, corresponds to crystalline domains that have melted during the annealing step and recrystallized on cooling. The high melting temperature peak corresponds to the portion of crystals that remained in the crystalline phase during the annealing step and undergoes some re-organization process (probably thickening). The melting temperature of these crystals increases with respect to the nascent powder (412 k) and is found in a range between 414 and $415 \mathrm{k}$. It is apparent that with increasing 
annealing time, the low melting temperature peak becomes more pronounced and increases in area against the one with the higher melting temperature. It is anticipated that the melting process during the annealing step occurs by detachment of crystal stems - enthalpic relaxation process $[5,30]$. The successive detachments of stems are hindered due to entanglements present in amorphous regions connecting different crystalline domains. This is clearly observed when similar experiments are performed on a commercially available UHMWPE (synthesized using a Z-N catalyst). Hence, the area of low temperature peak normalized by the total area of the two peaks can give an indication on the distribution of entanglements in the amorphous region of the semi-crystalline polymer as obtained from the reactor. Subsequent to the first melting after the annealing step, cooling and the second heating run is performed. These results into the anticipated crystallization and melting temperatures at $392 \mathrm{~K}$ and $407 \mathrm{~K}$, respectively, for all the polymers evaluated. The evolution of the area as a function of the annealing time of Figure 6 is plotted in Figure 7 together with the characterization of the different polymers synthesized using different co-catalysts (and in combination with the BHT) and commercial entangled UHMWPE for comparison.

The initial slope and the highest value reached at longer annealing time are related to the entanglement density of the polymer. Higher values and steeper slopes correspond to a polymer with a lower entanglement density (for similar molecular weight). 


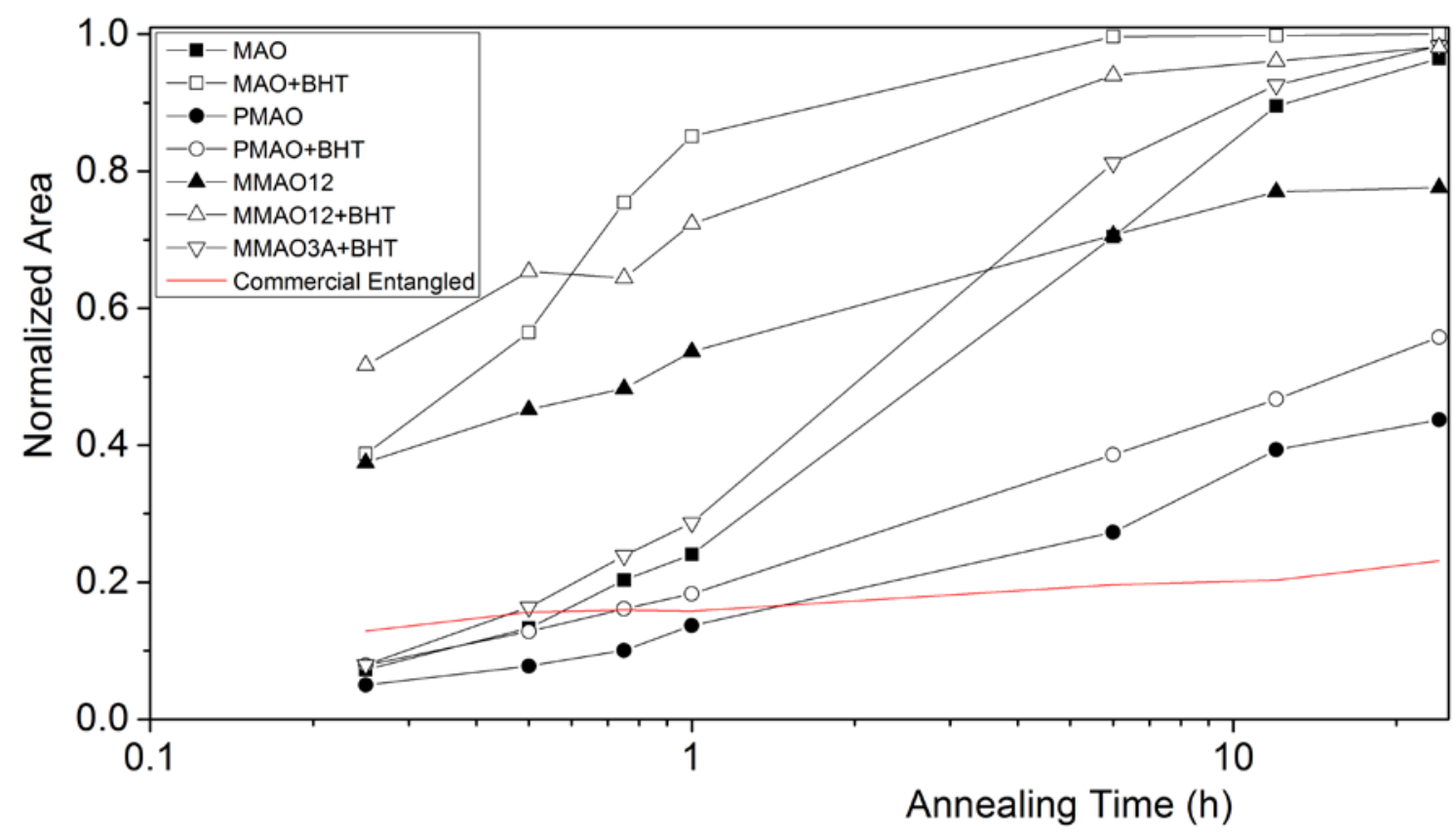

Figure 7 Evolution of the normalized area as a function of time for the polymers synthesized. Filled and unfilled squares correspond to the polymer synthesized using MAO and MAO+BHT respectively; filled and unfilled circles correspond to the polymers synthesized using $\mathrm{PMAO}, \mathrm{PMAO}+\mathrm{BHT}$ respectively; filled and unfilled up-triangles correspond to polymers synthesized using MMAO12 and MMAO12+BHT respectively; unfilled down-triangles corresponds to the polymers synthesized using MMAO3A+BHT; red line corresponds to commercial entangled polymer (Sigma Aldrich, $M_{\mathrm{w}} 3-6^{\star} 10^{6} \mathrm{~g} / \mathrm{mol}$ ).

All the polymers synthesized show an increase in the normalized area as a function of annealing time, which suggests that the synthesized polymers have a reduced number of entanglements, thus confirming the modulus build-up as observed in the rheological studies. It has to be noticed that whenever the BHT is used, the final value achieved is higher compared with the activation without BHT (Figure 8). However, the rheological analysis does not always match this small difference. According to previous findings [7], when the BHT is used with MAO, more chains with low entanglements are produced due to unlock of the dormant sites. 

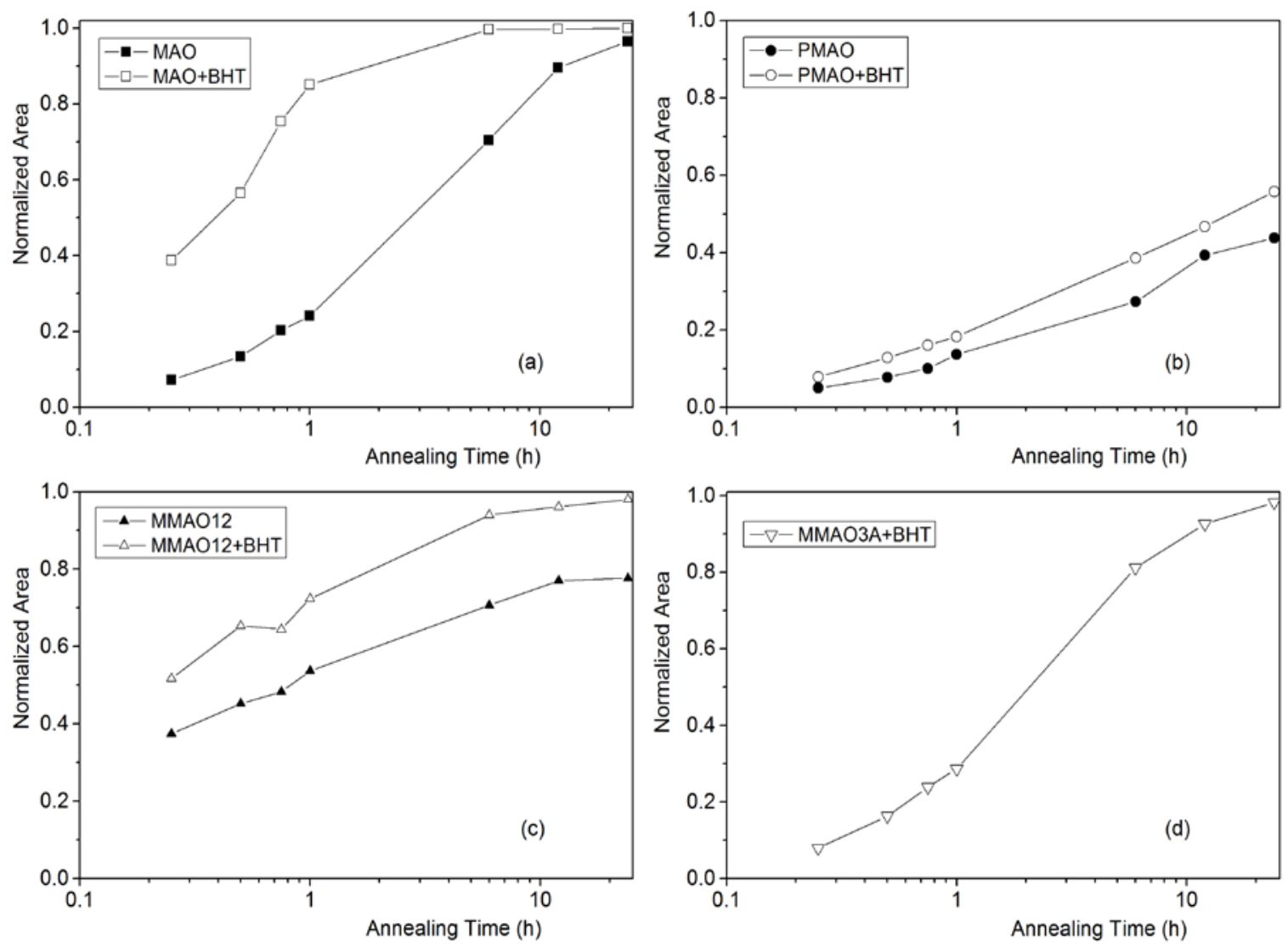

Figure 8 Evolution of the area ratio as a function of time for the polymers synthesized using MAO, MAO+BHT (a); PMAO, PMAO+BHT (b); MMAO12 and MMAO12+BHT (c); MMAO3A+BHT (d) .

In the case of the catalyst activation with PMAO and MMAO12 the same findings of Ref. [7] could be applied; a higher amount of chains with a low entanglement density are produced when the BHT is used in the activation. Hence, it is also confirmed by DSC experiments that the effect of the co-catalyst modifier is to produce chains with a lower number of entanglements also when the catalyst is activated with other aluminoxane co-catalysts beside MAO. Distinction in the melting during annealing is observed between the samples PMAO and MMAO12 and the others samples. These findings suggest that the samples synthesized in the presence of PMAO and MMAO12 are more entangled compared to the others. 


\section{$\underline{\text { Uniaxial Solid-State Deformation }}$}

Uniaxial solid-state deformation of the polymers synthesized using the cocatalysts MMAO12 and MMAO3A, both in combination with $\mathrm{BHT}$, have been performed. Both polymers show high draw ratio and relative low drawing tension compared with entangled commercial polymer which further supports the disentangled state of the synthesized polymer [2]. The high values of tensile modulus and tensile strength for both polymers are in agreement with the synthesis of UHMWPE having reduced amount of entanglements in the amorphous phase. Some difference in the values of tensile strength and modulus are observed for the polymer synthesized using MMAO3A+BHT as co-catalyst (Figure 9). The MMAO3A is sold as heptane solution, which after catalyst activation is injected in toluene (reaction medium). Possibly, the MMAO3A+catalyst in heptane form an emulsion in toluene leading to a high local catalyst concentration increasing the entanglement density. The polymer synthesized using MMAO12+BHT as co-catalyst shows higher values of tensile strength (4.3 N/tex, 4.2 GPa) and modulus (220 N/tex, $210 \mathrm{GPa}$ ). Similar values have been reported in our earlier work for the polymer synthesised using MAO as co-catalyst [2,5]. It is interesting to notice that all polymer characterization techniques (rheology, DSC and uniaxial solid-state deformation) used in this study show similarity in the results obtained from the polymers synthesized using MAO and MMAO12+BHT. 


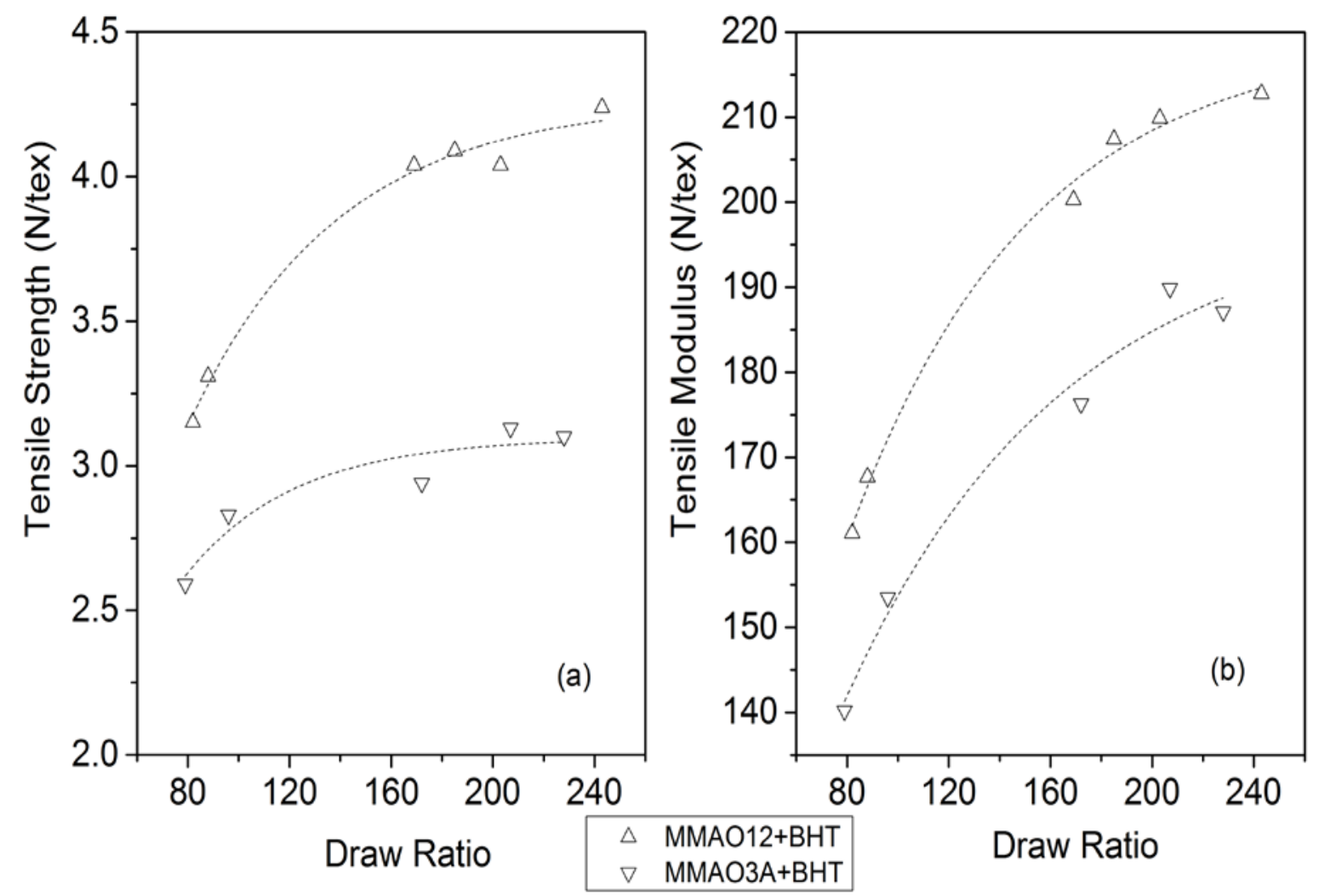

Figure 9 Tensile strength (a) and tensile modulus (b) as a function of draw ratio of the polymer synthesized using MMAO12+BHT (up-triangles) and MMAO3A+BHT (downtriangles). The lines have been drawn for visual guidance to the data points. These experiments were performed on the tapes obtained using the two set of samples. The nascent powder was converted into tapes following the protocol described in the experimental section and in reference [2].

\section{Conclusion}

We have reported the synthesis of UHMWPE with a reduced number of entanglements, using catalytic systems derived from the combination of the bis[N-(3tert-butylsalicylidene)pentafluoroanilinato] Titanium (IV) dichloride catalyst and different aluminoxane co-catalysts, the latter with and without a modifier, namely BHT. Molar mass and entangled state of the nascent powder samples are estimated using linear rheology. Regarding the entangled state of the polymers and DSC characterization methods are used to back up the rheological studies. Uniaxial solidstate deformations further support the DSC and rheological data. The results 
obtained in term of catalyst activity and molecular features of the polymer can lead to the following conclusions.

1) The FI catalyst can be successfully activated when using MAO, PMAO and MMAO12. The resulting active species are capable of synthesizing UHMWPE with $M_{\mathrm{w}}$ in a range of 5 million $\mathrm{g} / \mathrm{mol}$ up to 11 million $\mathrm{g} / \mathrm{mol}$. The catalytic system formed by the combination of $\mathrm{FI}$ catalyst+MMAO3A shows considerably depressed activity, probably due to the presence of TiBAl that modifies the catalyst [22].

2) On one hand, trapping of TMA by BHT increases the catalytic activity of systems activated using MAO, PMAO and MMAO12. On the other hand, in the case of MMAO3A, the effect of BHT is even more pronounced, suggesting that $\mathrm{BHT}$ is able to interact with both, TMA and TiBAl, protecting the catalyst from modification(s). This is confirmed by the observation that in the presence of BHT the PDI decreases. The two observations together suggest that in the presence of $\mathrm{BHT}$ a higher number of active sites is formed.

3) The choice of co-catalyst has no significant influence on the molecular weight of the polymer produced. However, the co-catalyst has an important role on the activation process where the amount of the PMAO, MMAO12 (both with and without $\mathrm{BHT}$ ) and MMAO3A+BHT required is higher than MAO to maintain the same catalyst activity, see Table 2 .

4) All polymers synthesized in this study show an elastic modulus build-up in melt, suggesting that the 'disentangled' character is achieved. Small difference in the entanglement densities are found when different co-catalysts are used. Also the co-catalyst modifier has a small effect on the entanglement density of the polymers produced. When the BHT is used, all the polymers synthesized have higher values of the normalized area at long annealing time compared with the activation without BHT; for some co-catalysts, also rheological measurements show a lower value of the starting point of the elastic modulus when the BHT is used. These results suggest that in the presence of BHT more chains having low entanglement density are formed. These findings are in accordance with our earlier report [7]. However, rheological determination of the starting point for these 
polymers is not always accurate enough to differentiate between differences of 0.1 $\mathrm{MPa}$. The polymers synthesized, due to their low entanglement density, could be processed in solid state leading to aligned fibers in tape geometry having high modulus/high strength (tensile strength and tensile modulus up to $4.3 \mathrm{~N} / \mathrm{tex},(4.2$ GPa) and modulus $220 \mathrm{~N} /$ tex (210 GPa) respectively).

\section{Acknowledgements}

The authors are grateful to Teijin Aramid and Loughborough University for the financial support. Niek Tops from Teijin Aramid is acknowledged for investigating the uniaxial deformation of the polymers.

[1] S. Rastogi, D.R. Lippits, G.W.M. Peters, R. Graf, Y. Yao, H.W. Spiess, Nature Materials 4 (2005) 635-641

[2] S. Rastogi; Y. Yao; S. Ronca; J. Bos; J. van der Eem, Macromolecules 44 (2011) 5558-5568

[3] a) R.S. Porter, T. Kanamoto, A.E. Zachariades, Polymer 35 (1994) 23 4979-4984 b) B.A. Griffin, Oceans '04. MTTS/IEEE Techno-Ocean '04 Conference 4 (2004) 1900-1903 c) A.S. Osaka, K.I. Kyoto, U.S. Patent 5,935,881 (1999) d) S.J. Bovenshen, J. Van Der Eem, S. Rastogi, J.A.W. Harings, A.A. Schaap, U.S. Patent WO2011076917 (2011)

[4] A. Pandey, Y. Champouret, S. Rastogi, Macromolecules 44 (2011) 4952-4960

[5] D. Romano, N. Tops, E. Andablo-Reyes, S. Ronca, S. Rastogi, Macromolecules, 47 (2014) 4750-4760

[6] S. Ronca, D. Romano, G. Forte, E. Andablo-Reyes, S. Rastogi, Adv. Polym. Sci. 31 (2012) 193-204

[7] D. Romano, E. A. Andablo-Reyes, S. Ronca, S. Rastogi, J. Polym. Sci. Pol. Chem. 51 (2013) 1630-1635

[8] H. Makio, H. Terao, A. Iwashita, T. Fujita, Chem. Rev. 111 (2011) 2363-2449

[9] M. Mitani, J. Mohri, Y. Yoshida, J. Saito, S. Ishii, K. Tsuru, S. Matsui, R. Furuyama, T. Nakano, H. Tanaka, S. Kojoh, T. Matsugi, N. Kashiwa, T. Fujita J. Am. Chem. Soc. 124 (2002) 3327-3336 
[10] E.Y.X. Chen, T.J. Marks, Chem. Rev. 100 (2000) 1391-1434

[11] H. Sin, W. Kaminsky, H.J. Vollmer, R. Woldt, Angew. Chem. Int. Ed. Engl. 19 (1980) 5

[12] H. Sinn, Macromol. Symp. 97 (1995) 27-52

[13] M.R. Mason, J.M. Smith, S.G. Bott, A.R. Barron, J. Am. Chem. Soc. 115 (1993) 4971-4984

[14] M. Ystenes, J.L. Eilertens, J. Liu, M. Ott, E. Rytter, J.A. Støvneng, J. Polym. Sci. Part A: Polym. Chem. 38 (2000) 3106-3127

[15] M. Bochman, Organometallics 29 (2010) 4711-4740

[16] E. Zurek, T. Ziegler, Organometallics 21 (2002) 83-92

[17] G.M. Smith, S.W.Palmaka, J.S.Rogers, D.B. Malpass, United State Patent 005831109A, November 3, (1998)

[18] J. Stellbrink, A. Niu, J. Allgaier, D. Richter, B.W. Koenig, R. Hartmann, G.W. Coates, L.J. Fetters, Macromolecules 40 (2007) 4972-7981

[19] From the MSDS obtained from www.akzonobel.com

[20] V. Busico, R. Cipullo, F. Cutillo, N. Friederichs, S. Ronca, B. Wang, J. Am. Chem. Soc. 125 (2003) 12402-12403

[21] T. Hasan, A. Ioku, K. Nishii, T. Shiono, T. Ikeda, Macromolecules 34 (2001) 3142-3145

[22] T. Li, F. W. Kong, R. Liu, Z.Y. Li, F. M. Zhu, Journal of Applied Polymer Science 119 (2011) 572-576

[23] D. Romano, S. Ronca, S. Rastogi, Macromolecular Symposia, To be published

[24] A. Pandey, A. Toda, S. Rastogi, Macromolecules 44 (2011) 8042-8055

[25] S. Talebi, R. Duchateau, S. Rastogi, J. Kaschta, G.W.M. Peters, P.J. Lemstra, Macromolecules 43 (2010) 2780-2788

[26] D.W. Mead, J. Rheol. 38 (1994) 1797-1827

[27] B. Wunderlich, Crystal Melting, Macromolecular Physics; Academic Press: New York, 3, 1980

[28] S.T. Milner, T.C.B. McLeish, Phys. Rev. Lett. 81 (1998) 725-728 
[29] E.A. Andablo-Reyes, E.L. de Boer, D. Romano, S. Rastogi, J. Rheol, 58 (2014) 1981-1991

[30] D.R. Lippits, S. Rastogi, G.W.H. Höhne, Phys. Rev. Lett. 96 (2006) 218-303 